\title{
Gastric tonometry in septic shock
}

\author{
M Hatherill, S M Tibby, R Evans, I A Murdoch
}

\begin{abstract}
Objectives-To investigate the prognostic value of intramucosal $\mathrm{pH}(\mathrm{pHi})$ and the relation among $\mathrm{pHi}$, arterial $\mathrm{pH}$, base excess, and lactate in children with septic shock.

Design-Children admitted to the paediatric intensive care unit with a diagnosis of septic shock were prospectively enrolled. A gastrointestinal tonometer (Tonometrics Division, Instrumentarium Corporation, Helsinki, Finland) was placed into the stomach and intramucosal pH, arterial pH, base deficit, and lactate were measured on admission and six hours later. Sequential data were analysed on 24 patients (17 survivors, seven nonsurvivors), median age 46 months (range: 2.8-168 months).

Results-Median pHi on admission was 7.39 (interquartile range 7.36-7.51) in survivors compared with 7.2 (interquartile range 7.18-7.35) in non-survivors ( $p=$ $0.01)$. There was no significant difference in arterial pH, base excess, or lactate among survivors and non-survivors. Admission pHi $<7.32$ predicted mortality with sensitivity (57\%), specificity (94\%), and positive predictive value $(80 \%)$. Patients with admission pHi $<7.32$ who failed to improve $\geqslant 7.32$ within six hours $(n=3)$ had $100 \%$ mortality.

Conclusion-In children with septic shock the admission pHi is significantly lower in non-survivors. pHi is a better prognostic indicator of mortality than either standard acid-base values or lactate. $\mathrm{pHi}<7.32$ that does not improve within six hours is associated with a poor prognosis. (Arch Dis Child 1998;78:155-158)
\end{abstract}

Keywords: tonometry; intramucosal $\mathrm{pH}$; septic shock

Septic shock is a major cause of death in the paediatric age group and the mortality rate remains high despite recent advances in paediatric intensive care. ${ }^{1}$ The need for accurate outcome prediction has focused attention on biochemical factors reflecting tissue hypoxia, such as lactate, acid-base variables, and arterial ketone body ratio as well as on global measurements of cardiac output which perform badly as prognostic indicators in children with septic shock. $^{23}$

Gastric tonometry has become routine in many adult intensive care units to monitor the course of haemodynamically unstable patients, as intramucosal acidosis may reflect a critical reduction of oxygen delivery to the hepatosplanchnic circulation, with the consequent risk of multiorgan failure (MOF) and death. ${ }^{4}$
This deterioration in intramucosal $\mathrm{pH}(\mathrm{pHi})$ often occurs before changes are detected in cardiac output or biochemical variables. However, there are few substantial reports of tonometry in children. In particular, there is a lack of data on the predictive value of $\mathrm{pHi}$ for mortality, and on the relation with other commonly used prognostic markers such as arterial $\mathrm{pH}$, base excess, and lactate..$^{5-8}$

We present the findings of a prospective observational study of gastrointestinal tonometry in children with septic shock.

\section{Patients and methods}

The study was approved by the hospital's ethics committee. Children between the ages of 1 month and 16 years admitted to our 16 bed paediatric intensive care unit (PICU) with a diagnosis of septic shock were enrolled over the period November 1995 to February 1997. Septic shock was defined according to the criteria of Saez-Llorens and McCracken as hypotension or poor capillary refill responding to fluid or pharmacological intervention in the presence of hyperthermia or hypothermia, tachycardia, tachypnoea, and either leucocytosis or leucopenia together with at least one of the following: acute mental changes, hypoxaemia, hyperlactataemia, and oliguria. ${ }^{9}$

Twenty six children were enrolled, of whom two (both survivors with normal $\mathrm{pHi}$ ) were excluded from analysis due to missing data points. The study group comprised 24 patients (17 survivors, seven non-survivors), median age 46 months (interquartile range 14.977.2)). All patients were mechanically ventilated and had been retrieved from referring hospitals by a PICU retrieval team, thus requiring resuscitation before transfer to the unit, including insertion of invasive monitoring lines and inotropic support. Diagnoses included meningococcal disease $(n=14)$, pneumococcal septicaemia $(n=1)$, staphylococcal septicaemia ( $n=1$ ), haemorrhagic shock encephalopathy ( $n=1$ ), and culture negative septic shock $(n=8){ }^{9}$

All children were paralysed and sedated at the time of enrolment. Patients received prophylactic sucralfate treatment until enteral feeding was attempted (usually at six hours) and continued if feeds were not tolerated. A 7.0 French (2.3 $\mathrm{mm}$ external diameter) gastrointestinal tonometer (Tonometrics Division, Instrumentarium Corporation, Helsinki, Finland) consisting of a blind ending tube with a carbon dioxide permeable balloon was placed into the stomach via either the nasal or oral route. Placement was occasionally aided by laryngoscopy and confirmed on the radiograph taken at admission. The semipermeable balloon was then filled with $2.5 \mathrm{ml}$ of saline 
Table 1 Age, PRISM score, risk of mortality, admission $p H i$, arterial $p H$, base excess, and lactate in the survivor and non-survivor groups. Data are expressed as median (interquartile range) and analysed by the Mann-Whitney test

\begin{tabular}{llll}
\hline & Survivors $(n=17,71 \%)$ & Non-survivors $(n=7,29 \%)$ & $p$ Value \\
\hline Age (months) & $37(15-64)$ & $65(30-108)$ & 0.38 \\
PRISM score & $11(9-17)$ & $17(10-21)$ & 0.30 \\
Risk of mortality & $0.07(0.03-0.18)$ & $0.12(0.05-0.36)$ & 0.70 \\
pHi & $7.39(7.36-7.51)$ & $7.20(7.18-7.35)$ & 0.01 \\
Arterial pH & $7.38(7.35-7.40)$ & $7.35(7.24-7.38)$ & 0.14 \\
Base excess & $-3.6(-5.4$ to -2.2$)$ & $-3.1(-4.7$ to -0.6$)$ & 0.73 \\
Lactate (mmol/l) & $1.4(0.9-1.9)$ & $1.8(1.3-2.4)$ & 0.34 \\
\hline
\end{tabular}

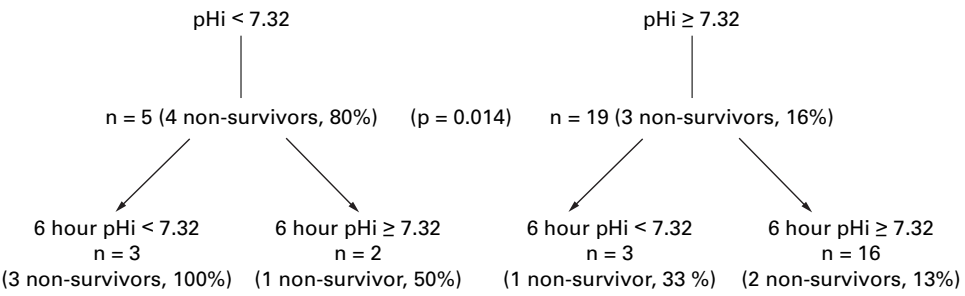

Figure 1 Mortality in children with septic shock: $p H i$ on admission and at six hours ( $n=$ 24) (seven non-survivors, 17 survivors).

and allowed to equilibrate for 60 minutes after which the saline was aspirated, the first $1 \mathrm{ml}$ discarded, and the remainder analysed for partial pressure of carbon dioxide $\left(\mathrm{PCO}_{2}\right) \cdot \mathrm{pHi}$ was calculated retrospectively from the tonometer saline $\mathrm{PCO}_{2}\left(\mathrm{TCO}_{2}\right)$ and the arterial bicarbonate concentrations according to the Henderson-Hasselbach equation: $\mathrm{pHi}=6.1+$ $\log \left[\left(\mathrm{HCO}_{3}\right) /\left(1.16 \times 0.03 \times \mathrm{TCO}_{2}\right)\right] .{ }^{10}$ The cut off value for abnormal $\mathrm{pHi}$ was derived from previous adult studies, 7.32 being the mean minus two SD. ${ }^{11} \mathrm{pHi}$ values were not used by attending clinicians to guide patient management.

A paediatric risk of mortality (PRISM) score and risk of mortality were calculated for each patient on the basis of physiological data over the first 24 hours, or in the case of nonsurvivors, a minimum of eight hours in the PICU. ${ }^{12}$ Baseline measurements of arterial $\mathrm{pH}$, base excess, and lactate were taken together with the $\mathrm{pHi}$ on admission to the unit.

Tonometer and arterial blood gas samples were analysed by the 1640 electrolyte blood gas analyser (Instrumentation Laboratories, Birchwood, UK). The same device was used for all samples. The coefficients of variation for this analyser are given as $6.4 \%$ and $13 \%$ for blood $\mathrm{PCO}_{2}$ and $\mathrm{pH}$. Lactate was measured using the YSI 2300 STAT plus analyser (Yellow Springs Instruments, Ohio, USA). All measurements were repeated six hours after admission and sequential data were analysed by the Fisher's exact test and Mann-Whitney $U$ test.

Table 2 Age, PRISM score, age specific risk of mortality, mortality, admission arterial $p H$, base excess, and lactate in children with normal $(\geqslant 7.32)$ and abnormal $(<7.32)$ pHi. Date are expressed as median (interquartile range) and analysed by the Mann-Whitney test except where indicated ( ${ }^{\star}$ Fisher's exact test)

\begin{tabular}{llll}
\hline & $p H i<7.32 n=5$ & $p H i \geqslant 7.32 n=19$ & $p$ Value \\
\hline Age (months) & $76(45-113)$ & $37(15-65)$ & 0.29 \\
PRISM score & $12(8-25)$ & $15(10-17)$ & 0.83 \\
Risk of mortality & $0.06(0.01-0.54)$ & $0.12(0.05-0.17)$ & 0.83 \\
Mortality (\%) & $4(80)$ & $3(16)$ & $0.01^{\star}$ \\
Arterial pH & $7.34(7.17-7.38)$ & $7.38(7.34-7.41)$ & 0.14 \\
Base excess & $-2.1(-7.7$ to-1.3) & $-3.6(-5.3$ to -2.3$)$ & 0.43 \\
Lactate (mmol/l) & $1.46(1.18-2.82)$ & $1.48(0.95-1.92)$ & 0.92 \\
\hline
\end{tabular}

\section{Results}

Admission $\mathrm{pHi}$ was lower in the seven non-survivors (median 7.2; interquartile range 7.18-7.35) compared with survivors (median 7.39; interquartile range 7.36-7.51) $(\mathrm{p}=$ 0.01 ). There were no significant differences in the age, PRISM score, risk of mortality, admission $\mathrm{pHi}$, arterial $\mathrm{pH}$, base excess, or lactate for the survivor and non-survivor groups (table 1). The seven deaths all occurred within five days (three within 24 hours) and were due to refractory shock alone $(n=2)$, refractory shock with acute lung injury $(\mathrm{n}=3)$, and shock with failure of three organs $(n=2)$.

Table 2 shows the age, PRISM score, risk of mortality, mortality, admission arterial $\mathrm{pH}$, base excess, and lactate concentrations for patients with normal $(\geqslant 7.32)$ and abnormal pHi (<7.32). The diagnostic case mix, timing, and mode of death was similar in both groups.

An admission $\mathrm{pHi}<7.32$ predicted mortality with sensitivity $57 \%$, specificity $94 \%$, and positive predictive value $80 \%(\mathrm{p}=0.01)$ (odds ratio $21.3 ; 95 \%$ confidence interval (CI) 1.7 to 263). Using a pHi value of 7.32 , patients could be subdivided into four groups according to their response to ongoing resuscitation. Those patients with an initial $\mathrm{pHi}<7.32$, who failed to improve $\geqslant 7.32$ within six hours $(n=3)$ had $100 \%$ mortality, although this difference did not reach statistical significance (fig 1). The $\mathrm{pHi}$ on admission was not closely associated with either lactate concentration $\left(r^{2}=0.27\right)$, arterial $\mathrm{pH}\left(r^{2}=0.35\right)$, or base excess $\left(r^{2}=0.02\right)$.

There were no failures of tonometer placement and no tonometer related complications.

\section{Discussion}

The development of gastric tonometry has provided physicians with a simple, low technology, minimally invasive method of evaluating perfusion of the hepatosplanchnic bed. Ischaemia of this vascular bed is thought to be an early event in the development of septic shock, MOF, and death. ${ }^{4}$

The technique of gastric tonometry was initially validated in animals, ${ }^{13}$ and increasing clinical use has shown the close association between low $\mathrm{pHi}$, morbidity, and mortality in adults. ${ }^{4114-16}$ Indeed, although adult studies have suggested that $\mathrm{pHi}$ is superior to conventional haemodynamic variables as a prognostic factor, ${ }^{11}$ use of this modality in paediatric practice has been restricted to a few centres. ${ }^{5}$

Krafte-Jacobs et al reported that in eight children with septic shock, $\mathrm{pHi}$ was significantly lower in the four non-survivors and correlated with lactate concentrations, but not with standard haemodynamic or oxygen derived variables. ${ }^{5}$ In a study of a heterogeneous group of 51 critically ill children, patients with an initial $\mathrm{pHi}<7.3$ had a $47 \%$ mortality rate. ${ }^{6}$ In that group $\mathrm{pHi}$ was found to be a better predictor of mortality than the PRISM score.

We aimed to evaluate the use of $\mathrm{pHi}$ as a prognostic variable in septic children, and to compare $\mathrm{pHi}$ with conventionally used indices of tissue oxygen debt. The study period included only the first six hours of admission to the PICU, as mortality from shock in the pae- 
diatric population follows a different clinical pattern from that of adults. Children with septicaemia often present in established MOF, and death usually occurs within 24 hours of admission to PICU, ${ }^{1}$ whereas critically ill adults tend to develop septic shock and MOF while already on the intensive care unit.

Our results agreed with those of adult workers who found that $\mathrm{pHi}$ was the best predictor of mortality when compared with conventional biochemical variables In particular, mortality was significantly greater among patients with pHi $<7.32$ on admission. ${ }^{14}{ }^{15}$ In our patients, admission $\mathrm{pHi}$ was significantly lower in non-survivors, with minimal overlap compared with the survivor group, and low $\mathrm{pHi}$ predicted death with a positive predictive value of $80 \%$ and odds ratio 21.3 (CI 1.7 to 263). This would suggest that abnormal pHi despite apparently adequate resuscitation is associated with a poor outcome. In contrast, neither arterial $\mathrm{pH}$, base excess, nor lactate was significantly different among survivors and nonsurvivors. The lack of a relation between lactate and mortality might be surprising, but as lactate production is affected by inhibition of pyruvate dehydrogenase in septicaemia, an increased lactate does not reflect tissue hypoperfusion alone. ${ }^{17}$

The superiority of pHi over the PRISM score as a predictor of mortality has been reported previously. ${ }^{6}$ All the children in our study group were resuscitated before transfer from the referring hospital by a dedicated PICU retrieval team, indeed the unexpectedly low calculated risk of mortality in our patients exposes a deficiency in this scoring system which has been noted by other authors ${ }^{18}$ - that is, that a PRISM score may be artificially lowered by resuscitation carried out before admission to the PICU. For this reason, many would argue that a scoring system based on pre-PICU data might better reflect the risk of mortality. ${ }^{19}$

Adult studies have shown that a persistently low pHi during the first 24 hours is associated with a worse prognosis ( $80 \%$ mortality). ${ }^{415}$ The fact that those few patients with persistently low pHi had $100 \%$ mortality, although this did not reach statistical significance, might suggest that those children with septic shock who have not resolved their mucosal acidosis six hours after initial resuscitation have a much worse prognosis. This would agree with the observation that many children with septic shock already have MOF on admission and require early intensive resuscitation, indeed three of the seven non-survivors died within 24 hours. Owing to our small sample size, larger studies will be necessary to determine whether failure to correct a low baseline $\mathrm{pHi}$ by six hours has true prognostic significance in paediatric septic shock.

Our results substantiate the hypothesis that low pHi may reveal a suboptimal response to resuscitation not detected by conventional haemodynamic monitoring. This additional therapeutic end point may enable treatment strategies specifically aimed at improving $\mathrm{pHi}$ to improve mortality in septic shock. ${ }^{20}$ However, it is not yet clear from adult experience whether $\mathrm{pHi}$ guided resuscitation has a future in paediatric practice. ${ }^{21}$

There is ongoing debate even in the adult literature concerning the assumptions inherent in the calculation of pHi. Firstly, how closely does the arterial bicarbonate, used in the Henderson-Hasselbach equation, reflect true intramucosal bicarbonate, and secondly, does the measured tonometer saline $\mathrm{PCO}_{2}$ accurately reflect intramucosal $\mathrm{PCO}_{2}$ ? $^{22}$ In order to eliminate potential sources of error in saline $\mathrm{PCO}_{2}$ measurement, some authors recommend either a phosphate buffered tonometer solution or a blood gas analyser specifically calibrated for measuring saline $\mathrm{PCO}_{2} \cdot{ }^{23}$ Finally, enteral feeding is also known to increase intraluminal $\mathrm{PCO}_{2}$, thereby producing spuriously low $\mathrm{pHi}$ values, and for this reason we collected tonometric data during the first six hours of admission only. ${ }^{24}$

Despite the potential limitations gastric tonometry is ideally suited to paediatric use as it provides a safe, minimally invasive, repeatable measure of hypoperfusion which has none of the risks associated with invasive haemodynamic monitoring. There were no complications of tonometer placement in any of the children in this study. While a tonometer may be sited in any child in whom a nasogastric or orogastric tube can be placed, to date paediatric studies of gastric tonometry have been limited by the size of the 7.0 French tonometers designed for adult sigmoid use. ${ }^{5-7}$ However, the development of 5.0 French $(1.7 \mathrm{~mm}$ external diameter) devices, specifically designed for paediatric practice, should make routine tonometric monitoring of sick children easier and more acceptable especially in the neonatal population.

\section{Conclusion}

Gastric tonometry offers a safe, minimally invasive technique of monitoring in children. Admission $\mathrm{pHi}$ in paediatric septic shock is significantly lower in non-survivors and is a better prognostic indicator of mortality than standard acid base variables or lactate concentrations. pHi $<7.32$ which fails to improve within six hours is associated with a poor prognosis.

1 Proulx F, Gauthier M, Nadeau D, et al. Timing and predictors of death in pediatric patients with multiple organ system failure. Crit Care Med 1994;22:1025-31.

2 Champion MP, Marsh MJ, Dalton RN, et al. Arterial or venous ketone body ratios in critically ill children. Intensive venous ketone body ratios
Care Med 1996;22:S196.

3 Pollack MM, Fields AI, Ruttiman UE. Distributions of cardiopulmonary variables in pediatric survivors and nonsurvivors of septic shock. Crit Care Med 1985;13:454-59.

4 Maynard N, Bihari D, Beale R, et al. Assessment of splanchnic oxygenation by gastric tonometry in patients with acute circulatory failure. $\mathscr{f} A M A$ 1993;270:1203-10.

5 Krafte-Jacobs B, Carver J, Wilkinson J. Comparison of gastric intramucosal $\mathrm{pH}$ and standard perfusional measurements in pediatric septic shock. Chest 1995;108:220-5.

6 Casado Flores J, Mora G, Garcia Teresa MA, et al. Prognostic value of the gastric intramucosal $\mathrm{pH}$ in mortality and multi-organ failure in critically ill children. Intensive Care Med 1996;22:S173.

7 Booker PD, Romer H, Franks R. Gut mucosal perfusion in neonates undergoing cardiopulmonary bypass. $\mathrm{Br} f$ Anaesth 1996;77:597-602.

8 Friedman G, Berlot G, Kahn RJ, et al. Combined measurements of blood lactate concentrations and gastric intramuments of blood lactate concentrations and gastric intramu-
cosal pH in patients with severe sepsis. Crit Care Med 1995; 23:1184-93. 
9 Saez-Llorens X, McCracken GH. Sepsis syndrome and septic shock in pediatrics: current concepts of terminology, pathophysiol

10 Trinder TJ, Lavery GG. The gastric tonometer. A valuable monitor of splanchnic perfusion? Anaesthesia 1996;51:16170 .

11 Fiddian-Green RG, Baker S. Predictive value of the stomach wall $\mathrm{pH}$ for complications after cardiac operations: comparison with other monitoring. Crit Care Med 1987;15:153-6.

12 Pollack MM, Ruttiman UE, Getson PR. Pediatric risk of mortality (PRISM) score. Crit Care Med 1988;16:1110-6.

13 Antonsson JB, Boyle CC, Kruithoff KL, et al. Validation of tonometric measurement of gut intramucosal $\mathrm{pH}$ during endotoxaemia and mesenteric occlusion in pigs. $\mathrm{Am} \mathcal{F}$ Physiol 1990;259:G519-23.

14 Trinder TJ, Lavery GG, Fee JPH, et al. Low gastric intramucosal pH: incidence and significance in intensive intramucosal pH: incidence and significance in inten

15 Gys T, Hubens A, Neels H, et al. Prognostic value of gastric intramural $\mathrm{pH}$ in surgical intensive care patients. Crit Care Med 1988;16:1222-4

16 Doglio GR, Pusajo JF, Egurrola MA, et al. Gastric mucosal $\mathrm{pH}$ as a prognostic index of mortality in critically il patients. Crit Care Med 1991;19:1037-40.
17 Vary TC, Siegel JH, Nakatani T, et al. Effect of sepsis on activity of pyruvate dehydrogenase complex in skeletal muscle and liver. Am $\mathcal{F}$ Physiol 1986;250:E634-40.

18 Britto J, Nadel S, Maconochie I, et al. Morbidity and severity of illness during interhospital transfer: impact of a specialised paediatric retrieval team. BMF 1995;311:8369.

19 Kanter RK, Edge WE, Caldwell CR, et al. Pediatric mortality probability estimated from pre-ICU severity of illness. Pediatrics 1997;99:59-63.

20 Maynard ND, Bihari DJ, Dalton RN, et al. Increasing splanchnic blood flow in the critically ill. Chest 1995;108: $1648-54$.

21 Gutierrez G, Palizas F, Doglio G, et al. Gastric intramucosal $\mathrm{pH}$ as a therapeutic index of tissue oxygenation in critically ill patients. Lancet 1992;339:195-9.

22 Russel JA. Gastric tonometry: does it work? Intensive Care Med 1997;23:3-6.

23 Knichwitz G, Kuhmann M, Brodner G, et al. Gastric tonometry: precision and reliability are improved by a phosphate buffered solution. Crit Care Med 1996;24:512-6.

24 Marik PE, Lorenzana A. Effect of tube feeding on the measurement of gastric intramucosal pH. Crit Care Med 1996;24:1498-1500. 